\title{
FREE RESOLUTIONS FOR MULTIGRADED MODULES: A GENERALIZATION OF TAYLOR'S CONSTRUCTION
}

\author{
Hara Charalambous and Alexandre Tchernev
}

\begin{abstract}
Let $Q=\mathbb{k}\left[x_{1}, \ldots, x_{n}\right]$ be a polynomial ring over a field $\mathbb{k}$ with the standard $\mathbb{N}^{n}$-grading. Let $\phi$ be a morphism of finite free $\mathbb{N}^{n}$-graded $Q$-modules. We translate to this setting several notions and constructions that appear originally in the context of monomial ideals. First, using a modification of the Buchsbaum-Rim complex, we construct a canonical complex $T_{\bullet}(\phi)$ of finite free $\mathbb{N}^{n}$-graded $Q$-modules that generalizes Taylor's resolution. This complex provides a free resolution for the cokernel $M$ of $\phi$ when $\phi$ satisfies certain rank criteria. We also introduce the Scarf complex of $\phi$, and a notion of "generic" morphism. Our main result is that the Scarf complex of $\phi$ is a minimal free resolution of $M$ when $\phi$ is minimal and generic. Finally, we introduce the LCM-lattice for $\phi$ and establish its significance in determining the minimal resolution of $M$.
\end{abstract}

\section{Introduction}

There is a plethora of significant papers examining free and minimal resolutions of monomial ideals. In contrast relatively little is known for $\mathbb{N}^{n}$-graded (multigraded) modules. Historically the prototype of a free resolution for monomial ideals is the Taylor resolution, [Ta60]. More recently [BaPeSt98] and [MiStYa00] give the minimal free resolution of generic monomial ideals, based on the idea of the Scarf complex; while [GaPeWe99] discuss the significance of the LCM-lattice in determining the minimal free resolution of an ideal. For multigraded modules, [ChDe01] discuss the second syzygies, while [Ya00] and [Mi00] among others generalize results concerning homological invariants of monomial ideals to multigraded modules.

In this paper we translate to the setting of morphisms of finite free multigraded modules several notions and constructions that appear originally in the context of monomial ideals. For a morphism $\phi$ of finite free multigraded modules, by using the formalism of what we decided to call Buchsbaum-Rim-Taylor systems, we construct canonical complexes $T_{\bullet}(\phi)$ and $S_{\bullet}(\phi)$ of finite free multigraded modules. The Taylor complex $T_{\bullet}(\phi)$ generalizes Taylor's resolution of a monomial ideal, and provides a free resolution for the cokernel $M$ of $\phi$ when certain rank criteria are satisfied for $\phi$. Just as the underlying linear algebra of the Taylor resolution is based on the Koszul complex, the linear algebra structure of the complex $T_{\bullet}(\phi)$ is based on the Buchsbaum-Rim complex [BuRi63]. The Scarf complex $S_{\bullet}(\phi)$ is a minimal subcomplex of the Taylor complex, and

Received September 1, 2002. 
appears to be the appropriate generalization of the Scarf complex of a monomial ideal.

We also introduce a notion of "generic" morphism. It is based on the combinatorial notion of generic monomial ideal of [BaPeSt98], together with the requirement that the morphism be sufficiently generic also from linear algebra point of view. Our main result, Theorem 5.6, is that the Scarf complex of $\phi$ is a minimal free resolution of $M$ when $\phi$ is a minimal presentation of $M$ and is a generic morphism. Finally, we introduce the LCM-lattice for a morphism $\phi$, and establish its significance in determining the minimal resolution of $M$.

The paper is organized as follows. In Section 2 we set our notation. For a morphism $\phi$ we introduce the coefficient matrix: a matrix with entries from $\mathbb{k}$ which determines the linear algebra structure of the resolution. We define certain submatrices of the coefficient matrix, and the kernel of a dual map which will turn out essential for the proof of the main theorem of this paper, Theorem 5.6.

In Section 3 we recall the construction of the Buchsbaum-Rim complex, presenting it in a form that guarantees that the differentials of the complex stay invariant under change of basis. This is important because, as it will be clear in Section 5, to compute the minimal resolution one has to consider a change of basis depending on the kernel of the dual map of Section 2 .

In Section 4 we introduce the notion of a BRT (Buchsbaum-Rim-Taylor) system, and use it to define a BRT complex for $\phi$. The BRT complex for the full BRT system is exact when the ranks of the submatrices of the coefficient matrix are high enough. This full BRT complex is the Taylor complex.

In Section 5 we introduce the LCM-lattice, the Scarf simplicial complex, and the notion of generic morphism. We describe the BRT system whose BRT complex is the Scarf complex and formulate our main result, Theorem 5.6.

Section 6 is devoted to the proof of Theorem 5.6.

Finally, in Section 7 we show how to extend to a certain class of morphisms (the morphisms of uniform rank) the arguments in [GaPeWe99] Theorem 3.3. This allows us to exhibit the role that the LCM-lattice plays in determining the structure of the minimal resolutions of these morphisms.

\section{Preliminaries}

Throughout this paper $\mathbb{k}$ is a field, and $Q=\mathbb{k}\left[x_{1}, \ldots, x_{n}\right]$ is the polynomial ring in $n$ variables over $\mathbb{k}$. Let $\alpha=\left(a_{1}, \ldots, a_{n}\right)$ be an element of $\mathbb{N}^{n}$. The support of $\alpha$ is the set $\operatorname{supp}(\alpha)=\left\{i \mid a_{i} \neq 0\right\}$. We write $x^{\alpha}$ for the monomial $x_{1}^{a_{1}} \ldots x_{n}^{a_{n}}$, and we set the degree of $x^{\alpha}$ to be $\left|x^{\alpha}\right|=\alpha$. This makes $Q$ into a $\mathbb{N}^{n}$-graded (or multigraded) algebra. We consider the partial order on $\mathbb{N}^{n}$ given by

$$
\alpha=\left(a_{1}, \ldots, a_{n}\right) \preceq \beta=\left(b_{1}, \ldots, b_{n}\right) \quad \Longleftrightarrow \quad a_{i} \leq b_{i} \text { for } i=1, \ldots, n .
$$

In addition, we define the join or $l \mathrm{~cm}$ of $\alpha$ and $\beta$ by

$$
\operatorname{lcm}(\alpha, \beta)=\alpha \vee \beta=\left(\max \left(a_{1}, b_{1}\right), \ldots, \max \left(a_{n}, b_{n}\right)\right) .
$$


The tensor product of multigraded $\mathbb{k}$-vector spaces is multigraded with $|x \otimes y|=$ $|x|+|y|$. Unadorned tensor products are over $\mathbb{k}$.

Let $E$ and $G$ be finite free multigraded $Q$-modules of ranks $e$ and $g$ respectively, and let $\phi: E \longrightarrow G$ be a multigraded morphism. We fix homogeneous bases $\epsilon_{1}, \ldots, \epsilon_{e}$ of $E$ and $\gamma_{1}, \ldots, \gamma_{g}$ of $G$, and we let $\Phi=\left(f_{i j}\right)$ be the matrix of $\phi$ in these bases. Thus the $j$ th column $\Phi_{j}$ of $\Phi$ gives the image of $\epsilon_{j}$ in $G$. We say that the degree of the column $\Phi_{j}$ is $\left|\epsilon_{j}\right|$.

To every such map $\phi$ we associate a map $s: U \longrightarrow W$ of vector spaces $U$ and $W$, where we let $U$ be the $\mathbb{k}$-vector space with basis $\epsilon_{1}, \ldots, \epsilon_{e}$ and let $W$ be the $\mathbb{k}$-vector space with basis $\gamma_{1}, \ldots, \gamma_{g}$ The matrix $C$ of the map $s$ in terms of the given bases is the coefficient matrix of $\Phi$ : each entry $f_{i j}$ of $\Phi$ is of the form $f_{i j}=c_{i j} x^{\alpha_{i j}}$ where $c_{i j} \in \mathbb{k}$, and $C=\left(c_{i j}\right)$. Clearly $\operatorname{rank} C=\operatorname{rank} \Phi=\operatorname{rank} \phi$.

Let $\alpha=\left(a_{1}, \ldots, a_{n}\right)$ be a multidegree. In Section 5 we will make use of the maps $s_{\alpha}$ and the vector spaces $V_{\alpha}$ and $K_{\alpha}$ which we define below. First, let $U_{\alpha}$ be the vector subspace of $U$ with basis those basis vectors of $U$ whose multidegree in $E$ is at most $\alpha$. Next, let $\Phi_{\alpha}$ be the submatrix of $\Phi$ on columns of degree $\preceq \alpha$, let $C_{\alpha}$ be the coefficient matrix of $\Phi_{\alpha}$, and let $s_{\alpha}$ be the restriction of the map $s$ to $U_{\alpha}$. The matrix of $s_{\alpha}$ is $C_{\alpha}$, and we write $V_{\alpha}$ for the image of $U_{\alpha}$ under $s$. Note that $U_{\alpha} \subseteq U_{\beta}$ and $V_{\alpha} \subseteq V_{\beta}$ when $\alpha \preceq \beta$.

\section{Remark 2.1.}

(a) The coefficient matrix $C$ is precisely the matrix obtained by removing the row and column labeling from the corresponding monomial matrix of $\phi$ in the sense of [Mi00].

(b) It is clear that the definitions of $U_{\alpha}, V_{\alpha}$, and $s_{\alpha}$ are independent of the choice of the homogeneous basis of $E$. In particular the definition of the map $s$ is independent of the choice of homogeneous bases of $E$ and $G$.

Let $I$ be a subset of $\{1, \ldots, e\}$. We write $U_{I}$ for the vector subspace of $U$ with basis $\left\{\epsilon_{i} \mid i \in I\right\}$. We call $V_{I}$ the image of $U_{I}$ under $s$, and denote by $s_{I}$ the restriction of $s$ to $U_{I}$.

For a $\mathbb{k}$-vector space $Z$ we set $Z^{*}=\operatorname{Hom}_{\mathbb{k}}(Z, \mathbb{k})$. If $V$ is the image of $s$ then the inclusions $V_{\alpha} \longrightarrow V$ and $V_{I} \longrightarrow V$ induce surjections $V^{*} \longrightarrow V_{\alpha}^{*}$ and $V^{*} \longrightarrow V_{I}^{*}$, and we write $K_{\alpha}$ and $K_{I}$ for the corresponding kernels. Note that if $v \in K_{\alpha}$ and $u \in U_{\alpha}$ then $v(s(u))=0$.

Finally, if $\mathbf{M}$ is a complex of vector spaces with $i$ th differential $\partial_{i}: M_{i} \longrightarrow$ $M_{i-1}$, then its shift $\mathbf{M}[k]$ is the complex with $M[k]_{i}=M_{i+k}$ and differential given by $\partial[k]_{i}=\partial_{k+i}$. We say that the complex $\mathbf{M}$ is exact if $\mathrm{H}_{i}(\mathbf{M})=0$ for $i \neq 0$. We say that $\mathbf{M}$ is split exact if it is exact, and $\mathrm{H}_{0}(\mathbf{M})=0$ as well.

\section{The Buchsbaum-Rim complex}

Let $s: U \longrightarrow W$ be a $\mathbb{k}$-vector space map. For the convenience of the reader, and to establish notation, we recall in this section the Buchsbaum-Rim complex of the map $s$, cf. [BuRi63], or [Ei95] Section A2.6.1. 
Let $V$ be a subspace of rank $r$ of $W$ such that $V$ contains the image of $s$. We recall that the $i$ th divided power $D_{i} V^{*}$ is the dual $\left(S_{i} V\right)^{*}$ of the $i$ th symmetric power $S_{i} V$, and refer to [Ei95] for the properties of these functors. For any integers $m, k \geq 0$ let $A_{\bullet}^{m, k}(s, V)$ be the complex

$$
0 \longrightarrow A_{e-k}^{m, k} \stackrel{\sigma_{e-k}^{m, k}}{\longrightarrow} A_{e-k-1}^{m, k} \stackrel{\sigma_{e-k-1}^{m, k}}{\longrightarrow} \cdots \stackrel{\sigma_{2}^{m, k}}{\longrightarrow} A_{1}^{m, k} \stackrel{\sigma_{1}^{m, k}}{\longrightarrow} A_{0}^{m, k} \longrightarrow 0
$$

where

$$
A_{i}^{m, k}=D_{m+i} V^{*} \otimes \wedge^{k+i} U
$$

The differential $\sigma_{i}^{m, k}$ of $A_{\bullet}^{m, k}$ is defined as the composition

$$
\begin{gathered}
D_{m+i} V^{*} \otimes \wedge^{k+i} U \\
\downarrow \delta \otimes \delta \\
D_{m+i-1} V^{*} \otimes V^{*} \otimes U \otimes \wedge^{k+i-1} U \\
\downarrow 1 \otimes \mu \circ(1 \otimes s) \otimes 1 \\
D_{m+i-1} V^{*} \otimes \wedge^{k+i-1} U,
\end{gathered}
$$

where $\delta$ is the diagonal map, and $\mu: V^{*} \otimes V \longrightarrow \mathbb{k}$ is the canonical pairing.

The complexes $A_{\bullet}^{m, k}(s, V)$ have been extensively studied. In the sequel we will use the following well known property.

Proposition 3.1. The complex $A_{\bullet}^{0, k}(s, V)$ is exact if and only if either $k \geq e$, or $V=\operatorname{Im}(s)$. The complex $A_{\bullet}^{m, 0}(s, V)$ is split exact if $m>0$ and $V=\operatorname{Im}(s)$.

Proof. The result is clear for $k \geq e$. Assume $0 \leq k<e$. Fixing a basis of $U$ provides an isomorphism $\wedge^{t} U \cong \wedge^{e-t} U^{*}$ and this identifies the complexes $A_{\bullet}^{0, k}(s, V)$ and $A_{\bullet}^{m, 0}(s, V)$ with the complexes $C^{k}$ and $C^{m+e}$ studied by Lebelt [Le73]. In particular, the proposition is an immediate consequence of [Le73], Corollary 1 to Theorem 5 and Corollary to Theorem 13; or see [Tc96], Theorem 4.1.

When $V$ is equal to the image of $s$ we write $A_{\bullet}^{k}(s)$ instead of $A_{\bullet}^{0, k}(s, V)$. In that case the induced by $s$ map $U \longrightarrow V$ is surjective, its dual $V^{*} \longrightarrow U^{*}$ is an inclusion, and we use it to identify $V^{*}$ as a subspace of $U^{*}$. It is now an elementary exercise in multilinear algebra to show that $\operatorname{Coker}\left(\sigma_{1}^{k}\right)$ is isomorphic to $\wedge^{e-k}\left(U^{*} / V^{*}\right)$ when $k \leq e$, and is 0 otherwise. In particular, we have

Corollary 3.2. If $k<r$ the complex $A_{\bullet}^{k}(s)$ is split exact. If $s$ is injective, then the complex $A_{\bullet}^{k}(s)$ is split exact for $k \neq e$.

In order to define the Buchsbaum-Rim complex, we splice together the complex $A_{\bullet}^{0, r+1}(s, V) \otimes \wedge^{r} V^{*}$ and the complex $U \stackrel{s}{\longrightarrow} W$, to obtain a diagram

$$
B_{\bullet}(s, V)=A_{\bullet}^{0, r+1}(s, V) \otimes \wedge^{r} V^{*} \stackrel{s_{2}}{\longrightarrow} U \stackrel{s}{\longrightarrow} W .
$$

Thus the complex $B_{\bullet}(s, V)$ has the form

$B_{\bullet}(s, V)=0 \longrightarrow B_{e-r+1} \stackrel{s_{e-r+1}}{\longrightarrow} B_{e-r} \stackrel{s_{e-r}}{\longrightarrow} \ldots \stackrel{s_{2}}{\longrightarrow} B_{1} \stackrel{s}{\longrightarrow} B_{0} \longrightarrow 0$ 
where $B_{0}=W$ and $B_{1}=U$, while $B_{i}=A_{i-2}^{r+1} \otimes \wedge^{r} V^{*}$ for $i \geq 2$. The splice map

$$
s_{2}: \wedge^{r+1} U \otimes \wedge^{r} V^{*} \longrightarrow U
$$

is defined as the composition

$$
\wedge^{r+1} U \otimes \wedge^{r} V^{*} \stackrel{\left(\left(1 \otimes \wedge^{r} s\right) \circ \delta\right) \otimes 1}{\longrightarrow} U \otimes \wedge^{r} V \otimes \wedge^{r} V^{*} \stackrel{1 \otimes \mu}{\longrightarrow} U \otimes \mathbb{k}=U,
$$

and for $i \geq 3$ we have $s_{i}=\sigma_{i-2}^{r+1} \otimes 1$. We note that the factor $\wedge^{r} V^{*}$ is just a copy of $\mathbb{k}$, but is needed in order to make the differentials of $B$. invariant under change of basis in $V$. We will have to consider such a change of basis in Section 5 .

Now that we have an invariant description of the differentials $s_{i}$, we can describe them in terms of basis elements as follows. Let $\epsilon_{1}, \ldots, \epsilon_{e}$ be a basis of $U$, let $\gamma_{1}, \ldots, \gamma_{g}$ be a basis of $W$, let $v_{1}, \ldots, v_{r}$ be a basis of $V^{*}$, and consider the dual basis on $V$. Let $C=\left(c_{i j}\right)$ be the matrix of the induced by $s$ map $U \longrightarrow V$ with respect to the above bases. Let $\beta=\left(b_{1}, \ldots, b_{r}\right)$ be a sequence of integers with $b_{1}+\cdots+b_{r}=p$. The elements of the form $v^{(\beta)}=v_{1}^{\left(b_{1}\right)} \ldots v_{r}^{\left(b_{r}\right)}$ where all the $b_{i}$ s are nonnegative are a basis for $D_{p} V^{*}$, and the element $v_{[r]}=v_{1} \wedge \cdots \wedge v_{r}$ forms a basis for $\wedge^{r} V^{*}$. Also, we set $v^{(\beta)}=0$ if $b_{i}<0$ for some $i$, and we write $\beta_{j}$ for the sequence $\beta_{j}=\left(b_{1}, \ldots, b_{j-1}, b_{j}-1, b_{j+1}, \ldots, b_{k}\right)$.

Similarly, the elements $\epsilon_{I}=\epsilon_{i_{1}} \wedge \cdots \wedge \epsilon_{i_{q}}$ (where the subset $I=\left\{i_{1}, \ldots, i_{q}\right\}$ with $i_{1}<\cdots<i_{q}$ ranges over all $q$-element subsets of $\left.\{1, \ldots, t\}\right)$ form a basis of $\wedge^{q} U$. Then we have for $i \geq 3$

$$
s_{i}\left(v^{(\beta)} \otimes \epsilon_{I} \otimes v_{[r]}\right)=\sum_{j=1}^{r} \sum_{l \in I} \operatorname{sgn}(l, I \backslash l) v_{j}\left(s\left(\epsilon_{l}\right)\right) v^{\left(\beta_{j}\right)} \otimes \epsilon_{I \backslash l} \otimes v_{[r]},
$$

while

$$
s_{2}\left(\epsilon_{J} \otimes v_{[r]}\right)=\sum_{l \in J} \operatorname{sgn}(l, J \backslash l) \operatorname{det}\left(C_{J \backslash l}\right) \epsilon_{l},
$$

where $|J|=r+1$. Note that if $V=W$ and $v_{1}, \ldots, v_{r}$ is the dual basis of $\gamma_{1}, \ldots, \gamma_{g}$ then the coefficients $v_{j}\left(s\left(\epsilon_{l}\right)\right)$ in the description of $s_{i}$ are just the entries $c_{j l}$.

We have the following well known property of the complex $B \cdot(s, V)$.

Proposition 3.3. When the rank $r$ of $V$ is greater than or equal to the rank e of $U$ the complex $B_{\bullet}(s, V)$ is exact if and only if the map $s$ is injective. When $r<e$ the complex $B_{\bullet}(s, V)$ is exact if and only if $V=\operatorname{Im}(s)$.

Proof. The proposition is immediate from Proposition 3.1, and [Ei95], Theorem A2.10.c.

When $V=\operatorname{Im}(s)$ we write $B_{\bullet}(s)$ instead of $B_{\bullet}(s, V)$. The exact complex $B_{\bullet}(s)$ is called the Buchsbaum-Rim complex of the map $s$.

Finally, we assign a multigrading on the components $B_{i}$ of the BuchsbaumRim complex $B_{\bullet}(s)$. The spaces $B_{0}=W$ and $B_{1}=U$ have the multigrading induced by the multidegrees of their basis elements $\gamma_{1}, \ldots, \gamma_{g}$ and $\epsilon_{1}, \ldots, \epsilon_{e}$, 
respectively. We set the multidegree of $\epsilon_{I}=\epsilon_{i_{1}} \wedge \cdots \wedge \epsilon_{i_{p}}$ in $\wedge^{p} U$ to be $\left|\epsilon_{I}\right|=$ $\operatorname{lcm}\left(\left|\epsilon_{i_{1}}\right|, \ldots,\left|\epsilon_{i_{p}}\right|\right)$, and thus obtain a multigrading on each of the vector spaces $\wedge^{p} U$. We also assign the multidegree 0 to all elements of $D_{i-2} V^{*}$ and $\wedge^{r} V^{*}$. This way we get a multigrading of $B_{i}=D_{i-2} V^{*} \otimes \wedge^{i+r-1} U \otimes \wedge^{r} V^{*}$ for $i \geq 2$.

\section{Buchsbaum-Rim-Taylor theory}

Let $s: U \longrightarrow W$ be the map associated to the multigraded map $\phi: E \longrightarrow G$ as described in Section 2, and let $r=\operatorname{rank} \phi$. In this section we introduce the notion of a BRT system and show how a BRT system gives rise to a BRT complex: a finite free complex of multigraded $Q$-modules. This allows us to generalize to the case of multigraded modules the Taylor resolution [Ta60].

Recall that $\Delta$ is the full simplex on the vertices $\{1, \ldots, e\}$.

Definition 4.1. A family of vector spaces $\mathbb{F}=\left\{F_{I}\right\}_{I \in \Delta}$ is a Buchsbaum-RimTaylor (BRT) system for the map $s$ if the following three conditions are satisfied:

1. $F_{I}=0$ whenever $|I| \leq r$;

2. $F_{I} \subseteq D_{|I|-r-1} V^{*}$ whenever $|I| \geq r+1$; and

3. $\mathbb{F}$ is $s$-compatible: whenever $|I|=p \geq r+2$, then

$$
s_{p-r+1}\left(F_{I} \otimes \epsilon_{I} \otimes \wedge^{r} V^{*}\right) \subseteq \bigoplus_{\substack{=p-1 \\ J \subset I}} F_{J} \otimes \epsilon_{J} \otimes \wedge^{r} V^{*} .
$$

Example 4.2. The main example of a BRT system is the full BRT system $\mathbb{F}^{\text {full }}$ where

$$
F_{I}= \begin{cases}D_{|I|-r-1} V^{*} & \text { if }|I| \geq r+1 \\ 0 & \text { otherwise. }\end{cases}
$$

The full BRT system is maximal, in the sense that it contains every other BRT system. In Section 5 we give another important example of a BRT system, the Scarf system.

Having a BRT system $\mathbb{F}$ allows us to construct a complex $R_{\bullet}(\mathbb{F}, \phi)$ of multigraded $Q$-modules as follows.

Definition 4.3. We set $R_{0}=Q \otimes B_{0}=G$, and $R_{1}=Q \otimes B_{1}=E$. For $i \geq 2$ we define the multigraded $Q$-module

$$
R_{i}=\bigoplus_{|I|=r+i-1} Q \otimes F_{I} \otimes \epsilon_{I} \otimes \wedge^{r} V^{*}
$$

We set $\phi_{1}=\phi$. For $i \geq 2$ we define the differentials $\phi_{i}: R_{i} \longrightarrow R_{i-1}$ by homogenizing the restrictions of the maps $s_{i}$ to the free modules specified by the BRT system. More precisely, if $z \in F_{I} \otimes \epsilon_{I} \otimes \wedge^{r} V^{*}$ and $y \in Q$, and if $s_{i}^{I, J}$ is the component of $s_{i}$ that sends $F_{I} \otimes \epsilon_{I} \otimes \wedge^{r} V^{*}$ to $F_{J} \otimes \epsilon_{J} \otimes \wedge \wedge^{r} V^{*}$, then the corresponding component $\phi_{i}^{I, J}$ of $\phi_{i}$ is

$$
\phi_{i}^{I, J}(y \otimes z)=x^{\left|\epsilon_{I}\right|-\left|\epsilon_{J}\right|} y \otimes s_{i}^{I, J}(z) .
$$


Since each map $\phi_{i}$ is obtained from the map $s_{i}$ by adjusting the multidegrees, and $B_{\bullet}(s)$ is a complex, it follows that

$R_{\bullet}(\mathbb{F}, \phi)=0 \longrightarrow R_{e-r+1} \stackrel{\phi_{e-r+1}}{\longrightarrow} R_{e-r} \longrightarrow \ldots \stackrel{\phi_{2}}{\longrightarrow} R_{1} \stackrel{\phi}{\longrightarrow} R_{0} \longrightarrow 0$

is also a complex. We call $R_{\bullet}(\mathbb{F}, \phi)$ the Buchsbaum-Rim-Taylor $(B R T)$ complex for the system $\mathbb{F}$ and the map $\phi$.

Definition 4.4. We write $T_{\bullet}(\phi)$ for the complex $R_{\bullet}\left(\mathbb{F}^{\text {full }}, \phi\right)$, where $\mathbb{F}^{\text {full }}$ is the full BRT system, and call it the Taylor complex of the map $\phi$.

Note that if $J$ is a monomial ideal and $\phi$ is the minimal presentation map for the module $Q / J$, then $T_{\bullet}(\phi)$ is precisely the Taylor resolution [Ta60] of $Q / J$.

We give a necessary and sufficient condition for the exactness of the complex $T_{\bullet}(\phi)$. For a multidegree $\alpha$ we say that the coefficient matrix $C_{\alpha}$ is of maximal rank if $\operatorname{rank} C_{\alpha}=\min \left(r, \operatorname{rank} U_{\alpha}\right)$, that is, if the rank of $C_{\alpha}$ is the smaller of the rank of $\phi$ and the number of columns of $C_{\alpha}$.

Theorem 4.5. The complex $T_{\bullet}(\phi)$ is exact if and only if for every multidegree $\alpha$ the matrix $C_{\alpha}$ is of maximal rank.

Proof. First we remark that a complex of multigraded $Q$-modules is exact if and only if it is exact in every multidegree $\alpha$.

Next we notice that the multihomogeneous element $x^{\nu} \otimes v^{(\beta)} \otimes \epsilon_{I} \otimes v_{[r]}$ in $R_{i}$ is of multidegree $\alpha$ if and only if the multidegree $\nu$ of the monomial $x^{\nu}$ added to the multidegree of $\epsilon_{I}$ equals $\alpha$. In other words, $\epsilon_{I}$ contributes to the component of multidegree $\alpha$ precisely when $\left|\epsilon_{i}\right| \leq \alpha$ for each $i \in I$. Therefore the component of $T_{\bullet}(\phi)$ of multidegree $\alpha$ is canonically isomorphic to the complex $B_{\bullet}\left(s_{\alpha}, V\right)$, where $V=\operatorname{Im}(s)$, therefore by Proposition 3.3 is exact if and only if the matrix $C_{\alpha}$ is of maximal rank.

Definition 4.6. We say that a multigraded morphism $\phi$ of rank $r$ is of uniform rank if all $g \times r$ submatrices of its coefficient matrix $C$ have rank equal to $r$.

The condition of Theorem 4.5 is of course guaranteed whenever $\phi$ is of uniform rank. Thus we have

Corollary 4.7. If $\phi$ is of uniform rank, then $T_{\bullet}(\phi)$ is exact.

Remark 4.8. The notion of uniform rank provides us with a precise description of what we mean when we say that a map $\phi$ is "sufficiently generic" from the point of view of linear algebra. Thus Corollary 4.7 states that if $\phi$ is sufficiently generic from the point of view of linear algebra, then the exactness of the Taylor complex does not depend on the combinatorial structure of $\phi$ and the choices on the multidegrees of the generators of $E$ and $G$.

We conclude this section with an example.

Example 4.9. Let $E=Q^{4}$ with standard basis $\epsilon_{1}, \ldots, \epsilon_{4}$ of multidegrees

$$
\left|\epsilon_{1}\right|=(3,0), \quad\left|\epsilon_{2}\right|=(2,1), \quad\left|\epsilon_{3}\right|=(1,2), \quad\left|\epsilon_{4}\right|=(0,3),
$$


and let $G=Q^{2}$ with standard basis of multidegrees $\left|\gamma_{1}\right|=(0,0)$, and $\left|\gamma_{2}\right|=$ $(1,0)$.

Let $\phi: E \longrightarrow G$ be the multigraded homomorphism with standard matrix

$$
\Phi=\left(\begin{array}{cccc}
x^{3} & x^{2} y & x y^{2} & y^{3} \\
x^{2} & 2 x y & 3 y^{2} & 0
\end{array}\right)
$$

Thus the coefficient matrix of $\phi$ is

$$
C=\left(\begin{array}{llll}
1 & 1 & 1 & 1 \\
1 & 2 & 3 & 0
\end{array}\right)
$$

we have $r=\operatorname{rank} \phi=\operatorname{rank} s=2$, and $V=\operatorname{Im} s=W$. For the basis $v_{1}, v_{2}$ of $V^{*}=W^{*}$ we choose the dual of the standard basis $\gamma_{1}, \gamma_{2}$ of $W$. Then the Taylor complex $T_{\bullet}(\phi)$ is

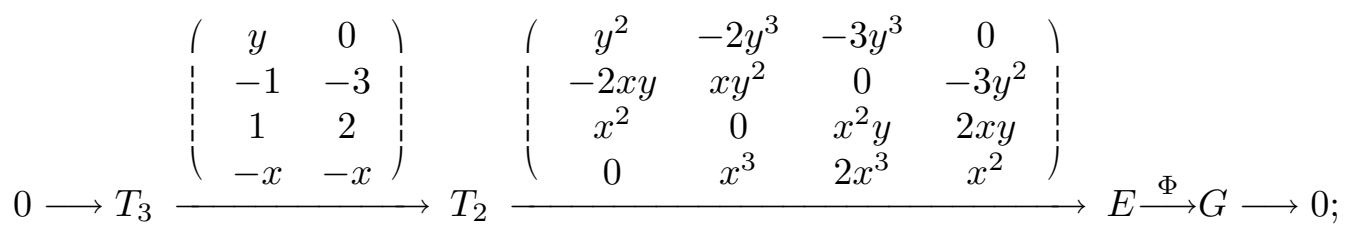

where the multidegrees of the generators of $T_{2}=Q \otimes \wedge^{3} U \otimes \wedge^{2} V^{*} \cong Q^{4}$ are

$$
\begin{aligned}
& \left|1 \otimes \epsilon_{\{1,2,3\}} \otimes v_{\{1,2\}}\right|=(3,2), \\
& \left|1 \otimes \epsilon_{\{1,2,4\}} \otimes v_{\{1,2\}}\right|=(3,3), \\
& \left|1 \otimes \epsilon_{\{1,3,4\}} \otimes v_{\{1,2\}}\right|=(3,3), \\
& \left|1 \otimes \epsilon_{\{2,3,4\}} \otimes v_{\{1,2\}}\right|=(2,3),
\end{aligned}
$$

and the multidegrees of the two generators of $T_{3}=Q \otimes V^{*} \otimes \wedge^{4} U \otimes \wedge^{2} V^{*} \cong Q^{2}$ are

$$
\begin{aligned}
& \left|1 \otimes v_{1} \otimes e_{\{1,2,3,4\}} \otimes v_{\{1,2\}}\right|=(3,3), \text { and } \\
& \left|1 \otimes v_{2} \otimes e_{\{1,2,3,4\}} \otimes v_{\{1,2\}}\right|=(3,3) .
\end{aligned}
$$

Since $\phi$ is clearly of uniform rank (all $2 \times 2$ minors of $C$ are non-zero), the complex $T_{\bullet}(\phi)$ is a resolution of $\operatorname{Coker}(\phi)$.

\section{The Scarf complex of a multigraded map}

In this section we introduce and study the notion of a generic multigraded map and construct the minimal resolution of the cokernel of a minimal generic multigraded map.

Definition 5.1. Let $\phi: E \longrightarrow G$ be a multigraded map.

1. The map $\phi$ is combinatorially generic if for every $1 \leq i<j \leq e$ the support of $\left|\epsilon_{i}\right|-\left|\epsilon_{j}\right|$ contains the supports of $\left|\epsilon_{i}\right|$ and $\left|\epsilon_{j}\right|$.

2. The map $\phi$ is generic if it is combinatorially generic and of uniform rank. 


\section{Remark 5.2.}

(a) The notion of a combinatorially generic map is a translation to maps of the notion of a generic monomial ideal [BaPeSt98]. When we deal with maps, we need to consider also the underlying linear algebra structure, hence the requirement that a generic map should be generic from both combinatorial and linear algebra point of view.

(b) In [MiStYa00] a notion of generic monomial ideal is defined which is more general than that of [BaPeSt98]. It is straightforward to use it to define a more inclusive notion of generic multigraded map. However, since at this point we do not know whether our main result, Theorem 5.6, holds with this more general definition of generic, we have elected not to pursue this line of investigation in this paper.

(c) By the deformation process of [BaPeSt98] every map of uniform rank can be deformed to a generic map. We will see in the remaining sections that several important homological properties of monomial ideals hold for maps of uniform rank.

Our next goal is to define the Scarf complex of a multigraded map $\phi$. This is a complex of multigraded free modules that is contained in the Taylor complex of $\phi$. It is a minimal complex when $\phi$ is minimal. In particular, it is contained in the minimal resolution of $M=\operatorname{Coker}(\phi)$ when $\phi$ is minimal and of uniform rank. The main result of this paper, Theorem 5.6, shows that the Scarf complex of $\phi$ is actually the minimal free resolution of $M$ when the map $\phi$ is minimal and generic.

We begin by translating to multigraded maps the notions of Scarf simplicial complex [BaPeSt98], and LCM-lattice [GaPeWe99].

Definition 5.3. Let $\phi: E \longrightarrow G$ be a multigraded map, and let $\epsilon_{1}, \ldots, \epsilon_{e}$ be a multihomogeneous basis of $E$.

1. The Scarf simplicial complex of $\phi$ is the subcomplex $\Delta_{S}=\Delta_{S}(\phi)$ of the full simplex $\Delta$ on the vertices $\{1, \ldots, e\}$ defined as:

$$
\Delta_{S}=\left\{I \in \Delta|| \epsilon_{I}|\neq| \epsilon_{J} \mid \text { for } J \neq I\right\} .
$$

2. The $L C M$-lattice $L_{\phi}$ of the map $\phi$ is the set of all elements of $\mathbb{N}^{n}$ that can be obtained as joins of some of the elements $\left|\epsilon_{1}\right|, \ldots,\left|\epsilon_{e}\right|$. Thus

$$
L_{\phi}=\left\{\alpha|\alpha=| \epsilon_{I} \mid \text { for some face } I \in \Delta\right\}
$$

is the set of those multidegrees that occur as multidegrees of faces of $\Delta$.

Let $\phi: E \longrightarrow G$ be a muiltigraded map of rank $r$, let $s: U \longrightarrow W$ be defined as in Section 2, and let $V=\operatorname{Im}(s)$. Before we define the Scarf system for $\phi$, we need to introduce some notation.

We partition the LCM-lattice $L_{\phi}$ into two subsets: the subset $L_{S}$ containing the multidegrees of the faces of $\Delta_{S}$, and its complement $L_{S}^{0}$. If $\alpha$ is a multidegree, we will denote by $I_{\alpha}$ the set of all indices $i$ for which $\left|\epsilon_{i}\right| \preceq \alpha$. Let $I(\alpha)$ be the 
intersection of all faces of $\Delta$ of degree $\alpha$, and set $I^{\alpha}=I_{\alpha} \backslash I(\alpha)$. Finally, recall from Section 2 that $K_{I^{\alpha}}$ is the kernel of the canonical surjection $V^{*} \longrightarrow V_{I^{\alpha}}^{*}$.

Definition 5.4. The Scarf system $\mathbb{F}_{S}$ for the map $\phi$ is the collection $\left\{F_{I}\right\}$ of vector spaces defined by:

$$
F_{I}= \begin{cases}0 & \text { if }|I| \leq r \\ D_{|I|-r-1} V^{*} & \text { if }|I| \geq r+1 \text { and } I \in \Delta_{S} \\ D_{|I|-r-1} K_{I^{\alpha}} & \text { if }|I| \geq r+1 \text { and } I=I_{\alpha} \text { for some } \alpha \in L_{S}^{0} \\ 0 & \text { otherwise. }\end{cases}
$$

By Proposition 5.7 the Scarf system is a BRT system, which allows for the following definition.

Definition 5.5. We write $S_{\bullet}(\phi)$ for the BRT complex associated with the Scarf system $\mathbb{F}_{S}$ and call it the Scarf complex of $\phi$.

The next theorem is the main result of this paper.

Theorem 5.6. Let $\phi: E \longrightarrow G$ be a minimal free multigraded presentation of a Noetherian multigraded $Q$-module $M$. If $\phi$ is generic, then the Scarf complex $S_{\bullet}(\phi)$ is a minimal free multigraded resolution of $M$ over $Q$.

We postpone the proof till the next section. We conclude this section with the proof that the Scarf system is a BRT system.

Proposition 5.7. The Scarf system is a BRT system.

Proof. We need to show that $\mathbb{F}_{S}$ is $s$-compatible. In other words if $I \in \Delta$ with $|I|=p \geq r+2$ we need to show that

$$
s_{p-r+1}\left(F_{I} \otimes \epsilon_{I} \otimes \wedge^{r} V^{*}\right) \subseteq \bigoplus_{\substack{|J|=p-1 \\ J \subset I}} F_{J} \otimes \epsilon_{J} \otimes \wedge^{r} V^{*}
$$

This is clear in all cases except when $I=I_{\alpha}$ for some $\alpha \in L_{S}^{0}$, so we assume this is the case. Thus $F_{I}=D_{p-r-1} K_{I^{\alpha}}$. Note that the component of $s_{p-r+1}$ in $F_{J} \otimes \epsilon_{J} \otimes \wedge^{r} V^{*}$ is zero when $\left|\epsilon_{J}\right|=\alpha$. Therefore, it will be enough to show that we have an inclusion

$$
D_{p-r-2} K_{I^{\alpha}} \subseteq F_{J}
$$

whenever $J \subset I$ with $J=p-1$, and $\left|\epsilon_{J}\right|=\beta \neq \alpha$. This is clear if $J \in \Delta_{S}$, so we assume that $J \notin \Delta_{S}$. Then $J \subseteq I_{\beta} \subsetneq I_{\alpha}=I$, hence $J=I_{\beta}$. Thus it will be enough to show that $K_{I^{\alpha}} \subseteq K_{I^{\beta}}$.

Let $L$ be a face of $I_{\beta}$ of degree $\beta$. Let $\{i\}=I_{\alpha} \backslash I_{\beta}$. Since $\left|\epsilon_{L}\right|=\beta$, we have that $\left|\epsilon_{L} \wedge \epsilon_{i}\right|=\alpha$. Thus $L \cup\{i\}$ contains $I(\alpha)$, hence $I(\alpha) \subseteq I(\beta) \cup\{i\}$. Since $I_{\beta}=I_{\alpha} \backslash\{i\}$, it follows that $I^{\beta} \subseteq I^{\alpha}$, and therefore $K_{I^{\alpha}} \subseteq K_{I^{\beta}}$, yielding the desired conclusion. 
Example 5.8. Let $\phi$ be the multigraded map of Example 4.9.

For the LCM-lattice of $\phi$ and its parts $L_{S}$ and $L_{S}^{0}$ we have

$$
\begin{aligned}
& L_{\phi}=\{(3,0),(2,1),(1,2),(0,3),(3,1),(3,2),(3,3),(2,2),(2,3),(1,3)\} ; \\
& L_{S}=\{(3,0),(2,1),(1,2),(0,3),(3,1),(2,2),(1,3)\} ; \\
& L_{S}^{0}=\{(3,2),(2,3),(3,3)\} .
\end{aligned}
$$

For the Scarf simplicial complex $\Delta_{S}$ we have

$$
\Delta_{S}=\{\{1\},\{2\},\{3\},\{4\},\{1,2\},\{2,3\},\{3,4\}\}
$$

For $\alpha \in L_{S}^{0}$ the faces $I_{\alpha}, I(\alpha)$, and $I^{\alpha}$ are

$$
\begin{array}{lll}
I_{(3,2)}=\{1,2,3\}, & I(3,2)=\{1,3\}, & I^{(3,2)}=\{2\} ; \\
I_{(2,3)}=\{2,3,4\}, & I(2,3)=\{2,4\}, & I^{(2,3)}=\{3\} ; \\
I_{(3,3)}=\{1,2,3,4\}, & I(3,3)=\{1,4\}, & I^{(3,3)}=\{2,3\} .
\end{array}
$$

For the spaces $K_{I^{\alpha}}$ with $\alpha \in L_{S}^{0}$ we obtain

$$
\begin{aligned}
& K_{I^{(3,2)}}=K_{\{2\}}=\operatorname{Ker}\left(V^{*} \longrightarrow V_{\{2\}}^{*}\right)=\mathbb{k} \cdot\left(2 v_{1}-v_{2}\right) ; \\
& K_{I^{(2,3)}}=K_{\{3\}}=\operatorname{Ker}\left(V^{*} \longrightarrow V_{\{3\}}^{*}\right)=\mathbb{k} \cdot\left(3 v_{1}-v_{3}\right) ; \\
& K_{I^{(3,3)}}=K_{\{2,3\}}=\operatorname{Ker}\left(V^{*} \longrightarrow V_{\{2,3\}}^{*}\right)=0 .
\end{aligned}
$$

For the spaces $F_{I}$ of the Scarf system for $\phi$ we obtain

$$
\begin{aligned}
& F_{\{1,2,3\}}=D_{0} K_{I^{(3,2)}}=D_{0} K_{\{2\}}=\mathbb{k} ; \\
& F_{\{2,3,4\}}=D_{0} K_{I^{(2,3)}}=D_{0} K_{\{3\}}=\mathbb{k} ; \\
& F_{\{1,2,3,4\}}=D_{1} K_{I^{(3,3)}}=K_{\{2,3\}}=0 ; \\
& F_{I}=0 \quad \text { otherwise. }
\end{aligned}
$$

Thus for the Scarf complex $S_{\bullet}(\phi)$ we obtain

$$
0 \longrightarrow S_{2} \stackrel{\left(\begin{array}{cc}
y^{2} & 0 \\
-2 x y & -3 y^{2} \\
x^{2} & 2 x y \\
0 & x^{2}
\end{array}\right)}{\longrightarrow} E \stackrel{ }{\longrightarrow} G \longrightarrow 0
$$

with $S_{0}=G \cong Q^{2}$, with $S_{1}=E \cong Q^{4}$, and with

$$
S_{2}=Q \otimes 1 \otimes \epsilon_{\{1,2,3\}} \otimes v_{\{1,2\}} \oplus Q \otimes 1 \otimes \epsilon_{\{2,3,4\}} \otimes v_{\{1,2\}} \cong Q^{2} .
$$

Since the map $\phi$ is generic, the Scarf complex is the minimal free resolution of $M=\operatorname{Coker}(\phi)$.

\section{The proof of Theorem 5.6}

Theorem 5.6 is an immediate consequence of the following slightly stronger statement.

Theorem 6.1. Let $\phi: E \longrightarrow G$ be a minimal multigraded presentation of a Noetherian multigraded $Q$-module $M$. If $\phi$ is combinatorially generic and the coefficient matrix $C_{\alpha}$ is of maximal rank for every multidegree $\alpha$, then the Scarf complex $S_{\bullet}(\phi)$ is the minimal free resolution of $M$. 
Proof. It is clear from the construction that the Scarf complex is minimal, hence it suffices to show that it is exact. Also, by construction $S_{\bullet}=S_{\bullet}(\phi)$ is a subcomplex of the Taylor complex $T_{\bullet}=T_{\bullet}(\phi)$, and $T_{\bullet}$ is exact by Theorem 4.5. Therefore it is enough to show that $X_{\bullet}=T_{\bullet} / S_{\bullet}$ is an exact complex. We will do this by showing that there is a filtration of $X_{\bullet}$ whose $i$ th quotient $Y_{\bullet}^{i}$ is the direct sum of exact complexes.

Note that the component of $X_{\bullet}$ in homological degree $m \geq 2$ is

$$
X_{m}=\bigoplus_{\substack{I \notin \Delta_{S} \\|I|=m+r-1}} Q \otimes H_{I} \otimes \epsilon_{I} \otimes \wedge^{r} V^{*}
$$

where

$$
H_{I}= \begin{cases}D_{m-2} V^{*} / D_{m-2} K_{I^{\alpha}} & \text { if } I=I_{\alpha} \text { for some } \alpha \in L_{S}^{0} ; \\ D_{m-2} V^{*} & \text { otherwise }\end{cases}
$$

and $X_{m}=0$ for $m \leq 1$.

We partition $L_{S}^{0}$ as follows. Let $L_{S}^{1}$ be the set of minimal multidegrees in $L_{S}^{0}$ (with respect to the partial order $\prec$ ). Once the sets $L_{S}^{1}, \ldots, L_{S}^{i}$ have been defined, we define $L_{S}^{i+1}$ to be the set of minimal elements in $L_{S}^{0} \backslash\left(L_{S}^{1} \cup \ldots \cup L_{S}^{i}\right)$. We also define $U L_{S}^{i}=L_{S}^{1} \cup \ldots \cup L_{S}^{i}$. Now define $X_{m}^{i} \subseteq X_{m}$ by

$$
X_{m}^{i}=\bigoplus_{\substack{I(\alpha) \subseteq I \subseteq I_{\alpha} \\|I|=m+r-1 ; \alpha \in U L_{S}^{i}}} Q \otimes H_{I} \otimes \epsilon_{I} \otimes \wedge^{r} V^{*} .
$$

It is straightforward that $X_{\bullet}^{i}$ is a subcomplex of $X_{\bullet}$ for each $i$, and that the quotient complex $Y_{\bullet}^{i}=X_{\bullet}^{i} / X_{\bullet}^{i-1}$ has as its component in homological degree $m \geq 2$ the module

$$
Y_{m}^{i}=\bigoplus_{\substack{I(\alpha) \subseteq I \subseteq I_{\alpha} \\|I|=m+r-1 ; \alpha \in L_{S}^{i}}} Q \otimes H_{I} \otimes \epsilon_{I} \otimes \wedge^{r} V^{*}
$$

while $Y_{m}^{i}=0$ for $m=0,1$. Thus to show that $S_{\bullet}$ is exact, it is enough to show that the complex $Y_{\bullet}^{i}$ is split exact for each $i \geq 1$. Note however that the complex $Y_{\bullet}^{i}$ decomposes into the direct sum of subcomplexes

$$
Y_{\bullet}^{i}=\bigoplus_{\alpha \in L_{S}^{i}} Y^{i}(\alpha)
$$

where the complex $Y^{i}(\alpha)$ has as its component in homological degree $m \geq 2$ the module

$$
Y^{i}(\alpha)_{m}=\bigoplus_{\substack{I(\alpha) \subseteq I \subseteq I_{\alpha} \\|I|=m+r-1}} Q \otimes H_{I} \otimes \epsilon_{I} \otimes \wedge^{r} V^{*},
$$

and $Y^{i}(\alpha)_{m}=0$ for $m=0,1$. Thus it suffices to show that each complex $Y^{i}(\alpha)$ is split exact. For the rest of this proof we fix $i$ and $\alpha \in L_{S}^{i}$, and we write $Z$ • for the complex $Y^{i}(\alpha)$. 
Since $\phi$ is combinatorially generic, if $\alpha \in L_{S}^{0}$, and $I$ and $J$ are such that $\left|\epsilon_{I}\right|=\left|\epsilon_{J}\right|=\alpha$, then $\left|\epsilon_{I \cap J}\right|=\alpha$ as well. Therefore there exists a unique minimal face $I(\alpha)$ of degree $\alpha$. Furthermore, a face $I$ has degree $\alpha$ if and only if we have $I(\alpha) \subseteq I \subseteq I_{\alpha}$, while the containment $I(\alpha) \subset I_{\alpha}$ is strict. So $i \in I_{\alpha} \backslash I(\alpha)$ if and only if $\left|\epsilon_{I_{\alpha} \backslash i}\right|=\left|\epsilon_{I_{\alpha}}\right|=\alpha$, and it follows that $I^{\alpha}=I_{\alpha} \backslash I(\alpha)=I_{\alpha^{\prime}}$ where $\alpha^{\prime}=\alpha-(1, \ldots, 1)$. Thus $K_{I^{\alpha}}=K_{I_{\alpha^{\prime}}}=K_{\alpha^{\prime}}$, and the short exact sequence of vector spaces

$$
0 \longrightarrow K_{\alpha^{\prime}} \longrightarrow V^{*} \longrightarrow V_{\alpha^{\prime}}^{*} \longrightarrow 0
$$

induces for $m \geq 2$ a canonical filtration

$$
0=T_{-1}^{m} \subseteq T_{0}^{m} \subseteq \cdots \subseteq T_{m-2}^{m}=D_{m-2} V^{*}
$$

on $D_{m-2} V^{*}$ whose $i$ th quotient $T_{i}^{m} / T_{i-1}^{m}$ is canonically isomorphic for $i \geq 0$ to $D_{m-2-i} K_{\alpha^{\prime}} \otimes D_{i} V_{\alpha^{\prime}}^{*}$. This way we obtain a filtration

$$
0=P_{m}^{-1}(I) \subseteq P_{m}^{0}(I) \subseteq P_{m}^{1}(I) \subseteq \cdots \subseteq P_{m}^{k}(I) \subseteq \cdots
$$

on each $H_{I}$, where for $i \geq 0$ we set

$$
P_{m}^{i}(I)= \begin{cases}T_{i}^{m} & \text { if } I \neq I_{\alpha} \\ T_{i}^{m} / T_{0}^{m} & \text { otherwise }\end{cases}
$$

When $i \geq 0$ it is clear that for the $i$ th quotient of this filtration we have canonically

$$
P_{m}^{i}(I) / P_{m}^{i-1}(I) \cong \begin{cases}0 & \text { if } I=I_{\alpha} \text { and } i=0 \\ D_{m-2-i} K_{\alpha^{\prime}} \otimes D_{i} V_{\alpha^{\prime}}^{*} & \text { otherwise. }\end{cases}
$$

Let $t=\left|I_{\alpha}\right|$. Set $Z_{m}^{-1}=0$, and for $k \geq 0$ define $Z_{m}^{k} \subseteq Z_{m}$ as

$$
Z_{m}^{k}=\bigoplus_{\substack{I(\alpha) \subseteq I \subseteq I_{\alpha} \\|I|=m+r-1}} Q \otimes P_{m}^{m+r+k-t}(I) \otimes \epsilon_{I} \otimes \wedge^{r} V^{*},
$$

It is straightforward from these definitions that $Z_{\bullet}^{k}$ is a subcomplex of $Z \bullet$ for each $k \geq-1$. Note that if $k \geq 0$ and $I=I_{\alpha}$ then $m+r+k-t=k+1 \geq 1$. Therefore for each $k \geq 0$ the quotient complex $\overline{Z_{\bullet}^{k}}=Z_{\bullet}^{k} / Z_{\bullet}^{k-1}$ has as its component in homological degree $m \geq 2$ the module

$$
\overline{Z_{m}^{k}}=\bigoplus_{\substack{I(\alpha) \subseteq I \subseteq I_{\alpha} \\|I|=m+r-1}} Q \otimes D_{t-r-k-2} K_{\alpha^{\prime}} \otimes D_{m+r+k-t} V_{\alpha^{\prime}}^{*} \otimes \epsilon_{I} \otimes \wedge^{r} V^{*},
$$

and is 0 in homological degrees $m=0,1$. Thus to complete the proof of the theorem it suffices to show that $\overline{Z_{\bullet}^{k}}$ is split exact.

Let $q=|I(\alpha)|$. We examine the differential of $\overline{Z_{\bullet}^{k}}$.

When $k>t-q-1$ it is clear that we have a canonical isomorphism of complexes

$$
\overline{Z_{\bullet}^{k}} \cong Q \otimes D_{t-r-k-2} K_{\alpha^{\prime}} \otimes A_{\bullet}^{q+k+1-t, 0}\left(s_{\alpha^{\prime}}\right)[-q+r-1] \otimes \epsilon_{I(\alpha)} \otimes \wedge^{r} V^{*} .
$$


By Proposition 3.1 the complex $A_{\bullet}^{q+k+1-t, 0}\left(s_{\alpha^{\prime}}\right)$ is split exact, therefore $\overline{Z_{\bullet}^{k}}$ is split exact as well.

Similarly when $k \leq t-q-1$ we have a canonical isomorphism of complexes

$$
\overline{Z_{\bullet}^{k}} \cong Q \otimes D_{t-r-k-2} K_{\alpha^{\prime}} \otimes A_{\bullet}^{0, t-q-k-1}\left(s_{\alpha^{\prime}}\right)[-p] \otimes \epsilon_{I(\alpha)} \otimes \wedge^{r} V^{*},
$$

where the shift $p$ is computed as $p=\max (q-r+1,2)$.

Next, recall that by assumption the rank $r_{\alpha^{\prime}}$ of $s_{\alpha^{\prime}}$ is equal to $\min \left(r,\left|I_{\alpha^{\prime}}\right|\right)$. If $\left|I_{\alpha^{\prime}}\right|=r_{\alpha^{\prime}} \leq r$ then $t-q-k-1=\left|I_{\alpha^{\prime}}\right|-k-1<r_{\alpha^{\prime}}$; thus $A_{\bullet}^{0, t-q-k-1}\left(s_{\alpha^{\prime}}\right)$ (hence also $\overline{Z_{\bullet}^{k}}$ ) is split exact by Corollary 3.2 .

Finally, assume $\left|I_{\alpha^{\prime}}\right|>r$. Then rank $s_{\alpha^{\prime}}=r$, hence $K_{\alpha^{\prime}}=0$. If $t-r-k-2 \neq 0$ then $D_{t-r-k-2} K_{\alpha^{\prime}}=0$, therefore $\overline{Z_{\bullet}^{k}}=0$ is split exact. If $t-r-k-2=0$ then

$$
t-q-k-1=r+2-q-1=r+1-|I(\alpha)|<r=r_{\alpha^{\prime}}
$$

where the last inequality follows by Lemma 6.2. Therefore $A^{0, t-q-k-1}\left(s_{\alpha^{\prime}}\right)$ (hence also $\overline{Z_{\bullet}^{k}}$ ) is split exact by Corollary 3.2

Lemma 6.2. With the assumptions of Theorem 6.1, if $\left|I_{\alpha^{\prime}}\right|>r$ then $|I(\alpha)| \geq 2$.

Proof. Suppose that $|I(\alpha)|=1$. Thus $I(\alpha)=\{l\}$ and $\left|e_{l}\right|=\alpha$. Since by asumption $C_{\alpha^{\prime}}$ is of maximal rank, we can choose $I^{\prime} \subset I_{\alpha^{\prime}}$ such that $\left|I^{\prime}\right|=r$ and rank $s_{I^{\prime}}=r$. Let $I=I^{\prime} \cup\{l\}$. Then $\phi_{2}\left(1 \otimes \epsilon_{I} \otimes v_{[r]}\right)$ is a minimal free generator of $E$, and is a syzygy of $\phi$. This contradicts the assumption that $\phi$ is a minimal presentation of the multigraded module $M$.

\section{The LCM-Lattice}

The importance of the LCM-lattice in determining the minimal resolutions of monomial ideals was exhibited in [GaPeWe99]. In the setting of multigraded maps one has to take into account also the underlying linear algebra structure. Our goal is to show that for maps of uniform rank, if the linear algebra structure is essentially the same then the structure of the minimal resolution is determined, subject to a certain compatibility condition, by the isomorphism class of the LCM-lattice.

Let $\phi: E \longrightarrow G$ be a multigraded map of finite free $Q$-modules, let $s: U \longrightarrow W$ be the associated map of vector spaces (see Section 2), let $V$ be the image of $s$, and let $L_{\phi}$ be the LCM-lattice. Similarly, let $Q^{\prime}$ be another polynomial ring, let $\phi^{\prime}: E^{\prime} \longrightarrow G^{\prime}$ be a multigraded map of finite free $Q^{\prime}$-modules, and consider the corresponding objects $s^{\prime}, V^{\prime}$, and $L_{\phi^{\prime}}$. Let $e$ be the rank of $E$, and let $e^{\prime}$ be the rank of $E^{\prime}$.

\section{Definition 7.1.}

(a) The maps $\phi$ and $\phi^{\prime}$ are called quasi-equivalent if there exists a choice of homogeneous bases for $E$ and $E^{\prime}$, and a choice of bases for $V$ and $V^{\prime}$ such that the matrices of the induced by $s$ and $s^{\prime}$ maps $U \longrightarrow V$ and $U^{\prime} \longrightarrow V^{\prime}$ are the same. 
(b) A choice of bases of $E, E^{\prime}, V$, and $V^{\prime}$ as in (a) is called a $Q E$-structure for the pair $\left(\phi, \phi^{\prime}\right)$.

(c) Assume $\phi$ and $\phi^{\prime}$ are quasi-equivalent. A function of sets $f: L_{\phi} \longrightarrow L_{\phi^{\prime}}$ is called $Q E$-compatible if there exist multihomogeneous bases $\epsilon_{1}, \ldots, \epsilon_{e}$ of $E$ and $\epsilon_{1}^{\prime}, \ldots, \epsilon_{e}^{\prime}$ of $E^{\prime}$ that are part of a QE-structure and satisfy $f\left(\left|\epsilon_{i}\right|\right)=\left|\epsilon_{i}^{\prime}\right|$ for all $i$.

To state our theorem, we need to introduce a slight generalization of the relabeling procedure of [GaPeWe99]. Let $T$ • be an extension of $\phi$ to a finite free multigraded complex of the form

$$
0 \longrightarrow T_{p} \stackrel{\phi_{p}}{\longrightarrow} T_{p-1} \longrightarrow \ldots \longrightarrow T_{2} \stackrel{\phi_{2}}{\longrightarrow} T_{1} \stackrel{\phi}{\longrightarrow} T_{0} \longrightarrow 0
$$

where $T_{0}=G$ and $T_{1}=E$; such that the free modules $T_{m}$ for $m \geq 1$ have generators with multidegrees in $L_{\phi}$. Let $f: L_{\phi} \longrightarrow L_{\phi^{\prime}}$ be a QE-compatible map which preserves joins that appear as multidegrees of minimal generators of the free modules $T_{m}$ for $m \geq 1$.

Using the map $f$ we relabel $T_{\bullet}$ in the spirit of [GaPeWe99], Construction 3.2. First, we replace $T_{0}$ with $G^{\prime}$, and $\phi$ with $\phi^{\prime}$. Next, if a free copy of $Q$ in $T_{m}$ for some $m \geq 1$ has multidegree $\alpha$ then after relabeling we get a free copy of $Q^{\prime}$ with multidegree $f(\alpha)$. Finally, we relabel the maps $\phi_{i}$ for $i \geq 2$ to $\phi_{i}^{\prime}=f\left(\phi_{i}\right)$ by homogenizing the images of $\phi_{i}$. We write $f\left(T_{\bullet}\right)$ for the resulting complex.

We are now ready to state the generalization of [GaPeWe99], Theorem 3.3.

Theorem 7.2. Let $\phi$ and $\phi^{\prime}$ be two quasi-equivalent multigraded maps of uniform rank $r$. Let $f: L_{\phi} \longrightarrow L_{\phi^{\prime}}$ be a $Q E$-compatible map which preserves joins of any $s$ atoms, where $s \geq r+1$. Let $F_{\bullet}(\phi)$ be the minimal resolution of the cokernel of the map $\phi$. Then $f\left(F_{\bullet}(\phi)\right)$ is a free resolution of the cokernel of $\phi^{\prime}$.

Proof. By Corollary 4.7, the Taylor complexes $T_{\bullet}(\phi)$ and $T_{\bullet}\left(\phi^{\prime}\right)$ resolve the cokernels of $\phi$ and $\phi^{\prime}$. Since $f\left(T_{\bullet}(\phi)\right)=T_{\bullet}\left(\phi^{\prime}\right)$, the zeroth homology of $f\left(T_{\bullet}(\phi)\right)$ is equal to the cokernel of $\phi^{\prime}$. Because $T_{\bullet}(\phi)=F_{\bullet}(\phi) \oplus P_{\bullet}$ where $P_{\bullet}$ is a direct sum of split exact complexes of the form $0 \longrightarrow Q \longrightarrow Q \longrightarrow 0$, it follows that $f\left(T_{\bullet}(\phi)\right)=f\left(F_{\bullet}(\phi)\right) \oplus f\left(P_{\bullet}\right)$ where $f\left(P_{\bullet}\right)$ is the direct sum of split exact complexes of the form $0 \longrightarrow Q^{\prime} \longrightarrow Q^{\prime} \longrightarrow 0$.

The remarks made in [GaPeWe99], Example 3.4, hold for the obvious generalizations for multigraded maps. We finish this section with an example where we apply Theorem 7.2 .

Example 7.3. Let $Q^{\prime}=\mathbb{k}[u, v, w]$, and let $\phi^{\prime}:\left(Q^{\prime}\right)^{4} \longrightarrow\left(Q^{\prime}\right)^{2}$ be the multigraded homomorphism with standard matrix:

$$
\Phi^{\prime}=\left(\begin{array}{cccc}
u^{2} v & u v w & u^{2} w & u^{2} w^{2} \\
u v & 2 v w & 3 u w & 0
\end{array}\right) .
$$

The coefficient matrix of $\phi^{\prime}$ equals the coefficient matrix of $\phi$ of Examples 4.9 and 5.8, hence the maps $\phi$ and $\phi^{\prime}$ are quasi-equivalent. Note that $\phi^{\prime}$ is not a 
generic map. Define $f: L_{\phi} \longrightarrow L_{\phi^{\prime}}$ by

$$
\begin{array}{lll}
f(3,0)=(2,1,0), & f(2,1)=(1,1,1), & f(1,2)=(2,0,1), \\
f(0,3)=(2,0,2), & f(3,1)=(2,1,1), & f(3,2)=(2,1,1), \\
f(3,3)=(2,1,2), & f(2,2)=(2,1,1), & f(2,3)=(2,1,2), \\
& f(1,3)=(2,0,2) . &
\end{array}
$$

Then $f$ satisfies the conditions of Theorem 7.2, but is not an isomorphism of LCM-lattices. Thus a free resolution of the cokernel of $\phi^{\prime}$ can be obtained by applying Theorem 7.2 to the minimal resolution of Example 5.8:

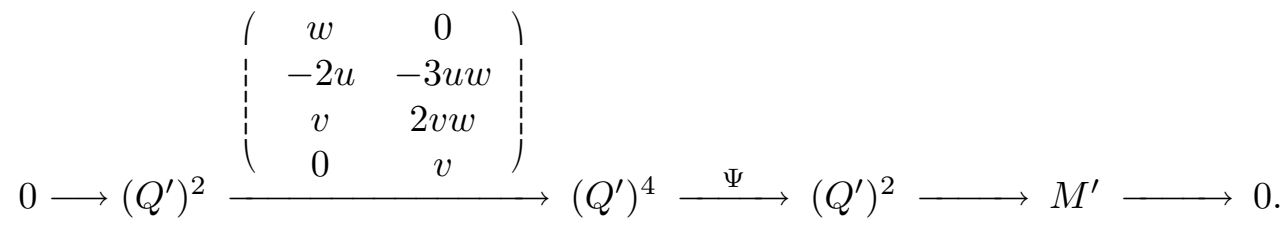

\section{References}

[BaPeSt98] D. Bayer, I. Peeva, B. Sturmfels, Monomial resolutions, Math. Res. Lett. 5 (1998), 31-46.

[BuRi63] D. Buchsbaum, D. Rim, A generalized Koszul complex, Bull. Amer. Math. Soc. 69 (1963), 382-385.

[ChDe01] H. Charalambous, C. Deno, Multigraded modules, New York J. Math. 7 (2001), $1-6$.

[Ei95] D. Eisenbud, Commutative algebra. With a view toward algebraic geometry, Graduate Texts in Mathematics, 150. Springer-Verlag, New York, 1995.

[GaPeWe99] V. Gasharov, I. Peeva, V. Welker, The lcm-lattice in monomial resolutions, Math. Res. Lett. 6 (1999), 521-532.

[Le73] K. Lebelt, Über Torsion äußerer Potenzen von Moduln der homologischen Dimension 1, Math. Ann. 211 (1974), 183-197.

[Mi00] E. Miller, The Alexander duality functors and local duality with monomial support, J. Algebra 231 (2000), 180-234.

[MiStYa00] E. Miller, B. Sturmfels, K. Yanagawa, Generic and cogeneric monomial ideals, Symbolic computation in algebra, analysis, and geometry (Berkeley, CA, 1998). J. Symbolic Comput. 29 (2000), 691-708.

[Ta60] D. Taylor, Ideals generated by monomials in an R-sequence, PhD Thesis, Univ. of Chicago, 1960.

[Tc96] A. Tchernev, Acyclicity of symmetric and exterior powers of complexes, J. Algebra 184 (1996), 1113-1135.

[Ya00] K. Yanagawa, Alexander duality for Stanley-Reisner rings and squarefree $\mathbb{N}^{n}$ graded modules, J. Algebra 225 (2000), 630-645.

Department of Mathematics, University at Albany, SUny, Albany, Ny 12222, U.S.A.

E-mail address: hara@math.albany.edu

E-mail address: tchernev@math.albany.edu 